antibodies (TTG) should be performed in patients with a diagnosis of lymphocytic duodenosis (LD), which may herald CD. The main objectives of this study were to determine the indications and histological findings for duodenal biopsies in a teaching hospital setting, and the prevalence of $\mathrm{LD}$ and new diagnoses of CD in this cohort.

Methods 1043 patients with duodenal biopsies taken during upper gastrointestinal endoscopy in a teaching hospital setting in 24 months (2009-11) were studied retrospectively. Demographics, indications and histological findings were documented. Patients with known CD were excluded from the study; if an individual had more than one biopsy in the time period then only the first biopsy was included. When a specific abnormality was targeted for biopsy this was recorded. For LD patients, TTG results were recorded where available.

Results The patients' median age was 60 (range 16-97); 55\% were female. Anaemia was the commonest indication for biopsy (in 51\% of patients) followed by weight loss and abdominal pain.

$76 \%$ of biopsies were normal. LD was found in 155 patients (15\%), of whom $64 \%$ were female. Biopsy appearances of CD with positive TTG were found in 13 patients (1.2\%). Documentation of serology was not always available and so the incidence of CD in this population may be underestimated. Crohn's disease was diagnosed in 3 biopsies (0.29\%). Graft versus host disease, drug effect, lymphangiectasia and infection were seen (1 Giardia, 1 atypical mycobacteria, 1 Strongyloides).

70 duodenal biopsies (7\%) targeted specific abnormalities, including 6 malignancies ( 3 adenocarcinoma, 1 carcinoid tumour and 1 lymphoma) and 6 benign polyps including 2 adenomas. Of the 973 non-targeted biopsies, 772 (79\%) were normal.

There were no statistical differences between positive biopsy results and specific indications.

Conclusion Whilst the majority of biopsies were normal, significant abnormalities were found in $24 \%$ and $\mathrm{LD}$ was present in $15 \%$. While important for the diagnosis of $\mathrm{CD}$, duodenal biopsy was also vital for the diagnosis other pathologies including infection, Crohn's disease, drug effect and tumours.

Disclosure of Interest None Declared.

\section{PTH-184 PUSH ENTEROSCOPY LEADS TO A CHANGE IN DIAGNOSIS IN THE MAJORITY OF PATIENTS WITH POSITIVE COELIAC SEROLOGY AND NEGATIVE DUODENAL BIOPSY}

doi:10.1136/gutjnl-2013-304907.671

'R Willington, ${ }^{2} \mathrm{~V}$ Lashmar, ${ }^{3} \mathrm{~K}$ Benes, ${ }^{2} \mathrm{~K}$ Barnett, ${ }^{2} \mathrm{~S}$ Weaver, ${ }^{2} \mathrm{R}$ McCrudden, ${ }^{4} \mathrm{~T}$ Smith, ${ }^{2} \mathrm{C}$ Gordon, 5,6, ${ }^{*} \mathrm{P}$ Cilclitira, ${ }^{2,6} \mathrm{~S}$ McLaughlin. ${ }^{1} \mathrm{AMU}$, Southampton General Hospital, Southampton; ${ }^{2}$ Department of Gastroenterology; ${ }^{3}$ Department of Pathology, Royal Bournemouth Hospital, Bournemouth; ' $D$ Department of Gastroenterology, Southampton General Hospital, Southampton; ${ }^{5}$ Department of Gastroenterology, St Thomas' Hospital; ${ }^{6}$ Diabetes and Nutritional Sciences Division, King's College London, London, UK

Introduction Coeliac disease is associated with significant morbidity, and is an underdiagnosed condition with an estimated prevalence of $1 \%$ in the UK population. Current British Society of Gastroenterology Society guidelines classify individuals with positive coeliac serology and negative duodenal biopsy to latent coeliac disease and do not recommend further investigation or the initiation of a gluten free diet.

In our recent practise patients at our institution with a strong clinical suspicion of coeliac disease and negative duodenal biopsies following a gluten containing diet undergo a push enteroscopy with biopsies of the jejunum and duodenum and histological reassessment of the original duodenal biopsies before a diagnosis of latent coeliac disease is made.

We aimed to investigate whether push enteroscopy and histological reassessment increased the diagnostic yield of coeliac disease.
Methods We searched our prospective endoscopy database for all patients with positive EMA or TTG results but negative D2 biopsies, who had undergone enteroscopy for a possible diagnosis of coeliac disease since 2007. Data including serology, result of histological reassessment of duodenal biopsies and assessment of jejunal biopsies were recorded.

Results 13 patients were identified; 4 (31\%) were male, the median age was 45 (range 19-78). In all 13, EMA testing had been performed, of which 9 were positive $(69 \%)$. In 9 , TTG results were available, of which 9 were positive (100\%). Following review of the original duodenal biopsies by a second pathologist, 7 (54\%) of the 13 patients were re-classified to active coeliac disease; median Marsh grade of 1 (range $0-2$ )

Of the remaining 6 patients, 5 (83\%) had evidence of active coeliac disease on jejunal biopsy; median Marsh grade of 2 (range 0-3b). Review of the jejunal biopsies alone, without review of original duodenal biopsies, would have led to active coeliac disease being diagnosed in 12 (92\%) of 13 patients.

Conclusion In this small retrospective series of patients with positive coeliac serology and negative duodenal biopsies, repeat histological assessment and jejunal biopsy led to a change in diagnosis in $92 \%$ of patients. These findings are unlikely to be limited to our institution. Our findings support the recommendation that a diagnosis of latent coeliac disease should only be made following repeat histological assessment and enteroscopy with jejunal biopsy.

Disclosure of Interest None Declared.

\section{PTH-185 CAPSULE ENDOSCOPY AND OBSCURE GASTROINTESTINAL BLEEDING: EXPERIENCE FROM A TERTIARY CENTRE}

doi:10.1136/gutjnl-2013-304907.672

1."P S Sidhu, 'K Drew, 'M E McAlindon, ${ }^{1} \mathrm{R}$ Sidhu. 'Gastroenterology, Royal Hallamshire Hospital, Sheffield Teaching Hospitals, Sheffield, UK

Introduction Capsule endoscopy (CE) is the first line modality to investigate the small bowel (SB) in patients with obscure gastrointestinal bleeding (OGB). We investigated the largest cohort of patients to date with OGB who underwent CE.

Methods All patients referred for CE for OGB were included retrospectively. Data was collected for demographics, clinical indication (overt bleeding $(\mathrm{OB})$ or iron deficiency anaemia (IDA)) the presence of co-morbidity, medications including anticoagulation, diagnostic yield (DY) and subsequent follow up.

Results A total of 1324 patients were identified. The mean age was 60 years with females consisting of $55 \%$. The indication for CE was IDA in $73 \%(n=971)$ and OB in $27 \%$. Significant co-morbidities were present in $38 \%$ of patients. Whilst $8 \%$ of patients were on warfarin, $6 \%(n=84)$ were transfusion dependent. The cumulative number of lesions identified was 1086. The overall DY was $36 \%(n=473)$ for lesions deemed to be the source of OGB. On logistic regression, increasing age $(p<0.001)$ and transfusion dependence $(p=0.02)$ were associated with a DY with CE. The commonest diagnosis was angioectasia (AE) in $23 \%(n=302)$. Other SB findings included ulcers and erosions in $25 \%(\mathrm{n}=326)$, fresh blood without a definite source in $6 \%(\mathrm{n}=79)$ whilst tumours, polyps and crohns disease represented $2 \%$ each respectively. Miscellaneous findings in the SB included diverticulae (14), SB strictures (15), SB varices (9), dielaufoy (1) and others (28). There was no significant difference in the DY between those with IDA and $O B(p=0.35)$ and between the sexes $(p=0.44)$. Management was altered in $78 \%(n=367)$ of those with a positive DY, in the form of further procedures $(42 \%, n=153)$. Of these, $35 \%(n=53)$ had double balloon enteroscopy, $24 \%(n=36)$ had push enteroscopy, $23 \%(n=35)$ had repeat standard endoscopy, 17 patients were referred for surgery whilst 2 patients had intraoperative enteroscopy. $34 \%(n=52)$ of patients received argon 